\title{
Design, Synthesis and Evaluation of Antiproliferative Activity of New Benzimidazolehydrazones
}

\author{
Valentina Onnis ${ }^{1, *}$, Monica Demurtas ${ }^{1}$, Alessandro Deplano ${ }^{1}$, Gianfranco Balboni ${ }^{1}$, \\ Anna Baldisserotto ${ }^{2}$, Stefano Manfredini ${ }^{2}$, Salvatore Pacifico ${ }^{3}$, Sandra Liekens ${ }^{4}$ and \\ Jan Balzarini ${ }^{4}$ \\ 1 Dipartimento di Scienze della Vita e dell'Ambiente, Sezione di Scienze Farmaceutiche, \\ Farmacologiche e Nutraceutiche, Università degli Studi di Cagliari, Via Ospedale 72, Cagliari I-09124, Italy; \\ monicademurtas@tiscali.it (M.D.); deplano.a@gmail.com (A.D.); gbalboni@unica.it (G.B.) \\ 2 Dipartimento di Scienze della Vita e Biotecnologie, Università degli Studi di Ferrara, \\ Via Fossato di Mortara 17-19, Ferrara I-44121, Italy; bldnna@unife.it (A.B.) mv9@unife.it (S.M.) \\ 3 Dipartmento di Scienze Chimiche e Farmaceutiche, Università degli Studi di Ferrara, \\ Via Fossato di Mortara 17-19, Ferrara I-44121, Italy; salvatore.pacifico@unife.it \\ 4 KU Leuven-University of Leuven, Department of Microbiology and Immunology, \\ Rega Institute for Medical Research, Laboratory of Virology and Chemotherapy, Leuven B-3000, Belgium; \\ sandra.liekens@rega.kuleuven.be (S.L.); jan.balzarini@rega.kuleuven.be (J.B.) \\ * Correspondence: vonnis@unica.it; Tel.: +39-070-675-8632
}

Academic Editor: Jean Jacques Vanden Eynde

Received: 31 March 2016; Accepted: 25 April 2016; Published: 30 April 2016

\begin{abstract}
The synthesis and antiproliferative activity of new benzimidazole derivatives bearing an hydrazone mojety at the 2-position is described. The new $N^{\prime}$-(4-arylidene)- $1 H$-benzo[d]imidazole-2carbohydrazides were evaluated for their cytostatic activity toward the murine leukemia (L1210), human T-cell leukemia (CEM), human cervix carcinoma (HeLa) and human pancreas carcinoma cells (Mia Paca-2). A preliminary structure-activity relationship could be defined. Some of the compounds possess encouraging and consistent antiproliferative activity, having $\mathrm{IC}_{50}$ values in the low micromolar range.
\end{abstract}

Keywords: benzimidazoles; hydrazones; antiproliferative activity

\section{Introduction}

As recently reviewed [1], benzimidazole is a privileged structure in medicinal chemistry because of its broad range of biological activities. The benzimidazole ring is present in some clinically used drugs, such as proton pump inhibitors, antihelmintic compounds, the antiviral enviroxime and the antihistaminic astemizole, but it may also display antimycobacterial, antimicrobial, anticonvulsant, analgesic, anti-inflammatory, anti-diabetic, antiprotozoal, antipsychotic, antioxidant and antitumoral properties [1].

Several series of benzimidazole derivatives have shown antiproliferative activity. Moreover, benzimidazole-5-carboxylic acid derivatives induced cell death in leukemic cells [2]. The antiproliferative activity of benzimidazole derivatives has been correlated to multi-target kinase inhibition $[3,4]$. Furthermore, benzimidazole carbamates showed antitubulin activity [5,6] and benzimidazolecarbazole conjugates were described to stabilize human telomeric DNA and to inhibit telomerase and topoisomerase I in cancer cells [7,8]. Benzimidazole has also been reported as a new scaffold endowed with sirtuin 1 and 2 inhibitory activity. These compounds also showed cytotoxicity against the breast cancer cell lines MCF-7 and MDA-MB-468 [9,10].

Hydrazone is another biologically active pharmacophore group. $\quad N$-(1'-Naphthyl)-3,4,5trimethoxybenzohydrazide has been reported as a potential anti-leukemia agent acting as a 
microtubule destabilizer [11]. 2-N-Heteroaryl caffeine hydrazones demonstrated high activity toward T-lymphoblastic leukemia cells and inhibited both RNA and DNA synthesis and mitosis [12]. 4-(2-Fluorophenoxy)quinolineacylhydrazones showed excellent antiproliferative activity and c-Met kinase inhibitory activity [13]. Salicylaldehyde isonicotinoylhydrazone analogs behaved as iron chelators especially in MCF-7 breast adenocarcinoma cells and this was correlated to their cytotoxic activity [14], while salicylaldehyde 1-arylmethyl-3-aryl-1H-pyrazole-5-carbohydrazide hydrazone derivatives inhibited the growth of A549 lung cancer cells [15]. We have previously reported on 2-arylamino-6-trifluoromethyl-3-(hydrazinocarbonyl)pyridines [16] showing in vitro inhibitory activity against human tumour cell lines at low micromolar to nanomolar concentrations. Furthermore 4-(diethylamino)salycilaldehyde hydrazones showed potent cytotoxicity against human tumour cell lines but showed no toxicity in athymic nude mice [17]. Although the biological activity of benzimidazole and hydrazine structures has been well documented, an extensive literature search revealed very few efforts to combine these two important moieties in a single molecular scaffold. Few studies described the anti-inflammatory and antimicrobial activity of benzimidazole-5-carboxylate and its hydrazone derivatives [18] and the antiproliferative activity of N-1-benzoimidazole acetohydrazides against various tumor cell lines [19]. Therefore, in this explorative work, based on the antiproliferative activity of benzimidazole and hydrazone containing structures we decided to investigate, in a dualistic approach [20], new benzimidazole-hydrazone compounds, namely $N^{\prime}$-(4-arylidene)- $1 H$-benzo[ $\left.d\right]$ imidazole-2-carbohydrazides and investigated their antiproliferative activity.

\section{Results}

\subsection{Chemistry}

The target hydrazones 3-19 were synthesized as shown in Scheme 1. High yields of 1H-benzo[d]imidazole-2-carbohydrazide (2) were achieved upon refluxing for $3 \mathrm{~h}$ in an ethanolic solution of the corresponding ethyl ester $\mathbf{1}$ and hydrazine hydrate. Hydrazones 3-19 were obtained in good to excellent yield by coupling the hydrazide 2 with the appropriate hydroxyarylaldehydes in ethanol. All the newly synthesized compounds gave corrected analytical data. The IR and NMR spectral data are consistent with the assigned structure. According to the literature, the presence of a single downfield resonating (8.49-9.79 ppm) $\underline{\mathrm{CH}}=\mathrm{N}$ signal indicates the exclusive formation of E-isomers [21].<smiles>CCOC(=O)c1nc2ccccc2[nH]1</smiles>

1<smiles>CC(C)[C@H]1CC[C@H](C(=O)NN)Nc2ccccc21</smiles>

2

ii)<smiles>O=C(N/N=C/Br)c1nc2ccccc2[nH]1</smiles>

Scheme 1. Synthesis of hydrazones 3-19. Reagents and conditions: (i) $\mathrm{NH}_{2} \mathrm{NH}_{2} \cdot \mathrm{H}_{2} \mathrm{O}$, EtOH, reflux, 3 h; (ii) $\mathrm{ArCHO}, \mathrm{EtOH}$ reflux, $5 \mathrm{~h}$. 


\subsection{Antiproliferative Activity}

The synthesized hydrazones 3-19 were evaluated in vitro for their inhibitory effects on the proliferation of murine leukemia (L1210), human T-lymphoblastic leukemia (CEM), human cervix carcinoma (HeLa) and human pancreas carcinoma (Mia Paca-2) cells. The presence of a 2-hydroxyl group on the arylidene moiety favourably modulates antiproliferative activity (Table 1).

Table 1. Antiproliferative activity of $N^{\prime}$-(4-arylidene)- $1 H$-benzo[d]imidazole-2-carbohydrazides 3-19.

\begin{tabular}{cccccc}
\hline Compd. & Ar & \multicolumn{5}{c}{ IC $_{\mathbf{5 0}}(\boldsymbol{\mu M})^{\mathbf{a}}$} & \\
\hline & & $\mathbf{L 1 2 1 0}$ & $\mathbf{C E M}$ & HeLa & Mia-Paca-2 \\
$\mathbf{3}$ & 2-OH-phenyl & $5.7 \pm 0.9$ & $4.4 \pm 0.2$ & $12 \pm 0.7$ & $34 \pm 0.9$ \\
$\mathbf{4}$ & 3-OH-phenyl & $94 \pm 7$ & $98 \pm 10$ & $88 \pm 12.5$ & $>100$ \\
$\mathbf{5}$ & 4-OH-phenyl & $47 \pm 2$ & $85 \pm 0.01$ & $>100$ & $>100$ \\
$\mathbf{6}$ & 2,4-(OH)2-phenyl & $2.6 \pm 0.9$ & $2.6 \pm 1.0$ & $4.7 \pm 1.6$ & $21 \pm 12$ \\
$\mathbf{7}$ & 2,5-(OH)2-phenyl & $22 \pm 7$ & $4.9 \pm 1.2$ & $77 \pm 18$ & $>100$ \\
$\mathbf{8}$ & 2,3,4-(OH)3-phenyl & $47 \pm 7$ & $20 \pm 8$ & $97 \pm 28$ & $>100$ \\
$\mathbf{9}$ & 2,4,6-(OH)3-phenyl & $90 \pm 7$ & $>100$ & $>100$ & $>100$ \\
$\mathbf{1 0}$ & 2-OH-4-OMe-phenyl & $1.6 \pm 0.9$ & $0.98 \pm 0.02$ & $4.0 \pm 0.4$ & $6.3 \pm 3.2$ \\
$\mathbf{1 1}$ & 2-OH-3-OEt-phenyl & $5.9 \pm 2.4$ & $6.3 \pm 1.9$ & $22 \pm 0.02$ & $23 \pm 8$ \\
$\mathbf{1 2}$ & 3-OH-4-OMe-phenyl & $92 \pm 4$ & $57 \pm 3.7$ & $>100$ & $>100$ \\
$\mathbf{1 3}$ & 2-OH-4-N(Et)2-phenyl & $14 \pm 2$ & $4.8 \pm 0.8$ & $23 \pm 3$ & $40 \pm 6$ \\
$\mathbf{1 4}$ & 2-OH-5-Cl-phenyl & $7.4 \pm 2.5$ & $1.8 \pm 0.6$ & $4.8 \pm 0.9$ & $9.2 \pm 4.0$ \\
$\mathbf{1 5}$ & 2-OH-5-Br-phenyl & $5.0 \pm 2.8$ & $1.8 \pm 0.9$ & $4.9 \pm 0.4$ & $35 \pm 5$ \\
$\mathbf{1 6}$ & 2-OH-naphtyl & $2.9 \pm 1.3$ & $1.0 \pm 0.01$ & $2.5 \pm 1.4$ & $7.9 \pm 0.3$ \\
$\mathbf{1 7}$ & phenyl & $>250$ & $>250$ & $>250$ & $220 \pm 37$ \\
$\mathbf{1 8}$ & 4-OMe-phenyl & $>250$ & $>250$ & $>250$ & $>250$ \\
$\mathbf{1 9}$ & napht-1-yl & $240 \pm 13$ & $>250$ & $>250$ & $>250$ \\
\hline
\end{tabular}

${ }^{\mathrm{a}} \mathrm{IC}_{50}$ values (compound concentration that reduces cell growth by $50 \%$ ) are shown.

\section{Discussion}

Hydrazones 10 and 16 inhibited the growth of all tested cell lines with low $(<10 \mu \mathrm{M})$ micromolar $\mathrm{IC}_{50}$ values. The replacement of the 2-hydroxynaphthyl group of compound 16 with the 2-hydroxyphenyl resulted in the hydrazone 3 endowed with reduced antiproliferative activity against HeLa and Mia Paca-2 cells. The shift of the hydroxyl group from the 2-position to the 3- or 4-position led to poorly active compounds 4 and 5. The introduction of a second 4-hydroxyl group (compound 6) caused an increase in activity with respect to the 2-hydroxy analog 3, whereas the presence of the 2,5-dihydroxybenzylidene group (compound 7) led to a clear reduction in inhibitory activity as compared with compounds 4 and 6 . The introduction of a third hydroxyl group (compounds 8 and 9) resulted in reduction or loss of cytostatic activity. A comparison of substituent effects revealed that the introduction of a 2-hydroxy-5-halobenzylidene moiety led to compounds $\mathbf{1 4}$ and 15 endowed with a better antiproliferative effect as compared with 2,5-dihydroxybenzylidene and 2-hydroxybenzylidene analogs 7 and 4 . The hydrazone of 5-chlorosalicylaldehyde $\mathbf{1 4}$ showed antiproliferative activity at single digit micromolar $\mathrm{IC}_{50}$ values on all tumor cell lines, while the 5-bromo derivative 15 was less active on Mia Paca-2 cells. The introduction of the 2-hydroxy-4-methoxybenzylidene moiety (compound 10) caused an increase in activity as compared with 2,4-dihydroxybenzylidene and 2-hydroxybenzylidene analogs 6 and 3. A reduction in activity was produced by the shift of the ether group from the 4- to the 3-position (hydrazone 11), while the shift of the hydroxyl group from the 2- to the 3-position (compound 12) led to a drop in activity. The replacement of the 4-methoxy group with a 4-(diethylamino) group was tolerated although hydrazone $\mathbf{1 3}$ was less active as compared with hydrazones 3 and $\mathbf{6}$. The hydrazones 17-19 lacking hydroxyl groups are completely inactive.

The efficacy of any drug depends on its high oral bioavailability, so we assessed the potential bioavailability of the most active compounds, using the adsorption, distribution, metabolism 
and elimination (ADME) prediction method by molinspiration (http:/ /www.molinspiration.com/ cgi-bin/properties).

According to Lipinski's rule of five [22] a compound to become a successful drug candidate, should have a molecular weight $\leqslant 500$, a $\log p \leqslant 5$, hydrogen bond donor sites $\leqslant 5$ and hydrogen bond acceptor sites ( $\mathrm{N}$ and $\mathrm{O}$ atoms) $\leqslant 10$. Predictions of the ADME properties for studied compounds (Table 2) showed that all the active compounds fulfilled this rule, similarly to clinically used drugs. Furthermore, the number of rotable bonds is important for conformational flexibility of the molecule. The total polar surface area (TPSA) is another key property that has been linked to drug bioavailability. These two parameters, i.e., the number of rotable bonds in a molecule $\leqslant 10$ and polar surface area $\leqslant 140 \AA$ are essential for a good oral bioavailability [23]. Theoretically, all hydrazones 3, 6-8, 10, 11, 13-16 should present good passive oral absorption and differences in their bioactivity cannot be attributed to this property.

Table 2. In silico physico-chemical properties (ADME) of active compounds 3, 6-8, 10, 11, 13-16.

\begin{tabular}{ccccccccc}
\hline Compd. & TPSA & n-ROTB & MV & MW & miLogP & n-ON & n-OHNH & n-Viol \\
\hline $\mathbf{3}$ & 90.37 & 3 & 242.94 & 280.29 & 2.64 & 6 & 3 & 0 \\
$\mathbf{6}$ & 110.60 & 3 & 250.96 & 296.29 & 2.13 & 7 & 4 & 0 \\
$\mathbf{7}$ & 110.60 & 3 & 250.96 & 296.29 & 2.13 & 7 & 4 & 0 \\
$\mathbf{8}$ & 130.83 & 3 & 258.97 & 312.29 & 1.67 & 8 & 5 & 0 \\
$\mathbf{1 0}$ & 99.61 & 4 & 268.48 & 310.31 & 2.677 & 7 & 3 & 0 \\
$\mathbf{1 1}$ & 99.61 & 5 & 285.29 & 324.34 & 2.62 & 7 & 3 & 0 \\
$\mathbf{1 3}$ & 93.61 & 6 & 322.45 & 351.41 & 3.47 & 7 & 3 & 0 \\
$\mathbf{1 4}$ & 90.37 & 3 & 256.47 & 314.73 & 3.29 & 6 & 3 & 0 \\
$\mathbf{1 5}$ & 90.37 & 3 & 260.82 & 359.18 & 3.42 & 6 & 3 & 0 \\
$\mathbf{1 6}$ & 90.37 & 3 & 286.93 & 330.35 & 3.79 & 6 & 3 & 0 \\
\hline
\end{tabular}

TPSA: topological polar surface area; n-ROTB: number of rotable bonds; MV: molecular volume; MW: molecular weight; milogP: logarithm of partition coefficient between $n$-octanol and water; n-ON: number of hydrogen bond acceptors; $n-O H N H$ : number of hydrogen bond donors; $n$-Viol: number of violations of Lipinski's rule.

\section{Materials and Methods}

\subsection{General Information}

All commercially available solvents and reagents were used without further purification. ${ }^{1} \mathrm{H}$ - and ${ }^{13} \mathrm{C}-\mathrm{NMR}$ spectra were recorded on an Inova 500 spectrometer (Varian, Palo Alto, CA, USA). The chemical shifts $(\delta)$ are reported in part per million downfield from tetramethylsilane (TMS), which was used as internal standard, and the spectra were recorded in hexadeuteriodimethylsulphoxide (DMSO- $d_{6}$ ). Infrared spectra were recorded on a Vector 22 spectrometer (Bruker, Bremen, Germany) in Nujol mulls. The main bands are given in $\mathrm{cm}^{-1}$. Positive-ion electrospray ionization (ESI) mass spectra were recorded on a double-focusing MAT 95 instrument (Finnigan, Waltham, MA, USA) with BE geometry. Melting points (mp) were determined on a SMP1 Melting Point apparatus (Stuart Scientific, Stone, UK) and are uncorrected. All products reported showed ${ }^{1} \mathrm{H}$ NMR spectra in agreement with the assigned structures. The purity of the tested compounds was determined by combustion elemental analyses conducted by the Microanalytical Laboratory of the Chemistry Department of the University of Ferrara with a MT-5 CHN recorder elemental analyzer (Yanagimoto, Kyoto, Japan) and the values found were within $0.4 \%$ of theoretical values. Hydrazones 17 and 18 were synthesized as previously described [24].

\subsection{Synthesis}

\subsection{1. $1 H$-benzo[d]imidazole-2-carbohydrazide (2)}

A mixture of ethyl $1 \mathrm{H}$-benzo[d]imidazole-2-carboxylate $(\mathbf{1}, 3.80 \mathrm{~g}, 20 \mathrm{mmol})$, and hydrazine monohydrate $(3 \mathrm{~mL}, 61.5 \mathrm{mmol})$ in $\mathrm{EtOH}(5 \mathrm{~mL})$ was refluxed for $3 \mathrm{~h}$. After cooling the formed 
precipitate was filtered off, washed with water $(5 \times 10 \mathrm{~mL})$ dried and used without further purification. Yield 80\%. Mp 240-242 ${ }^{\circ} \mathrm{C}$ (lit. [25] 217-219). IR: 3321, 3265, 3066, 1661, $1609 \mathrm{~cm}^{-1} .{ }^{1} \mathrm{H}-\mathrm{NMR}: \delta 4.62$ $\left(\mathrm{s}, 2 \mathrm{H}, \mathrm{NH}_{2}\right), 7.28(\mathrm{~m}, 2 \mathrm{H}, \mathrm{Ar}), 7.53(\mathrm{~d}, J=8.0 \mathrm{~Hz}, 1 \mathrm{H}, \mathrm{Ar}), 7.70(\mathrm{~d}, J=8.0 \mathrm{~Hz}, 1 \mathrm{H}, \mathrm{Ar}), 10.14(\mathrm{~s}, 1 \mathrm{H}, \mathrm{NH})$, $13.20(\mathrm{~s}, 1 \mathrm{H}, \mathrm{NH})$. ESI-MS $m / z 177(\mathrm{M}+\mathrm{H})^{+}$. Anal. Calcd for $\mathrm{C}_{8} \mathrm{H}_{8} \mathrm{~N}_{4} \mathrm{O}: \mathrm{C}, 54.54 ; \mathrm{H}, 4.58 ; \mathrm{N}, 31.80$. Found: $\mathrm{C}, 54.57 ; \mathrm{H}, 4.57 ; \mathrm{N}, 31.76$.

\subsubsection{General Procedure for the Synthesis of Hydrazones 3-19}

A mixture of hydrazide 2 ( $1 \mathrm{mmol})$ and the appropriate aldehyde $(1 \mathrm{mmol})$ in $\mathrm{EtOH}(10 \mathrm{~mL})$ was refluxed for $5 \mathrm{~h}$. After cooling the formed precipitate was filtered off and purified by crystallization from the adequate solvent to give the hydrazone derivatives.

(E)-N'-(2-Hydroxybenzylidene)-1H-benzo[d]imidazole-2-carbohydrazide (3). Yield 78\%. Mp > $250{ }^{\circ} \mathrm{C}(\mathrm{EtOH})$. IR: 3191, 1664, 1614, $1556 \mathrm{~cm}^{-1} .{ }^{1} \mathrm{H}-\mathrm{NMR}: \delta 6.94$ (m, 2H, Ar), 7.31 (m, 3H, Ar) 7.67 (m, 3H, Ar), 8.83 $(\mathrm{s}, 1 \mathrm{H}, \mathrm{CH}), 11.23(\mathrm{~s}, 1 \mathrm{H}, \mathrm{OH}), 12.78(\mathrm{~s}, 1 \mathrm{H}, \mathrm{NH}), 13.51$ (s, 1H, NH). ${ }^{13} \mathrm{C}-\mathrm{NMR}: \delta$ 105.4, 109.9, 115.1, $115.9,123.0,126.8,129.7,136.3,135.2,138.4,141.2,145.9,150.1$. ESI-MS $m / z 281(\mathrm{M}+\mathrm{H})^{+}$. Anal. Calcd for $\mathrm{C}_{15} \mathrm{H}_{12} \mathrm{~N}_{4} \mathrm{O}_{2}$ : C, 64.28; $\mathrm{H}, 4.32 ; \mathrm{N}, 19.99$. Found: C, 64.22; H, 4.33; N, 20.05.

(E)-N'-(3-Hydroxybenzylidene)-1H-benzo[d]imidazole-2-carbohydrazide (4). Yield 78\%. Mp > $250{ }^{\circ} \mathrm{C}(\mathrm{EtOH})$. IR: 3221, 1677, 1610, $1576 \mathrm{~cm}^{-1} .{ }^{1} \mathrm{H}-\mathrm{NMR}: \delta 6.85(\mathrm{~d}, J=8.0 \mathrm{~Hz}, 1 \mathrm{H}, \mathrm{Ar}), 7.11(\mathrm{~d}, J=7.5 \mathrm{~Hz}, 1 \mathrm{H}, \mathrm{Ar})$, $7.21(\mathrm{~s}, 1 \mathrm{H}, \mathrm{Ar}), 7.26(\mathrm{~d}, J=7.5 \mathrm{~Hz}, 1 \mathrm{H}, \mathrm{Ar}), 7.29-7.78(\mathrm{~m}, 4 \mathrm{H}, \mathrm{Ar}), 8.56(\mathrm{~s}, 1 \mathrm{H}, \mathrm{CH}), 9.69(\mathrm{~s}, 1 \mathrm{H}, \mathrm{OH})$, 12.40 (s, 1H, NH), 13.47 (s, 1H, NH). ${ }^{13} \mathrm{C}-\mathrm{NMR}: \delta 105.3,105,4,109.8,117.1,117.9,123.1,130.1,129.8$, 138.7, 138.8, 144.3, 149.9, 150.2. ESI-MS $m / z 281(\mathrm{M}+\mathrm{H})^{+}$. Anal. Calcd for $\mathrm{C}_{15} \mathrm{H}_{12} \mathrm{~N}_{4} \mathrm{O}_{2}: \mathrm{C}, 64.28 ; \mathrm{H}$, 4.32; N, 19.99. Found: C, 64.34; H, 4.31; N, 20.03.

(E)- $\mathrm{N}^{\prime}-(4-H y d r o x y b e n z y l i d e n e)-1 H$-benzo[d]imidazole-2-carbohydrazide (5). Yield $80 \% . \mathrm{Mp}>250{ }^{\circ} \mathrm{C}$ (1-PrOH). IR: 3289, 1668,1609, $1584 \mathrm{~cm}^{-1} .{ }^{1} \mathrm{H}-\mathrm{NMR}: \delta 6.86$ (d, $\left.J=8.5 \mathrm{~Hz}, 2 \mathrm{H}, \mathrm{Ar}\right), 7.34(\mathrm{~d}, J=7.0 \mathrm{~Hz}$, 2H, Ar), 7.57-7.59 (m, 3H, Ar), $7.77(\mathrm{~d}, J=7.0 \mathrm{~Hz}, 1 \mathrm{H}, \mathrm{Ar}), 8.53(\mathrm{~s}, 1 \mathrm{H}, \mathrm{CH}), 10.03(\mathrm{~s}, 1 \mathrm{H}, \mathrm{OH}), 12.23$ (s, 1H, NH), 13.43 (s, 1H, NH). ${ }^{13} \mathrm{C}-\mathrm{NMR}: \delta 105.8,106,3,116.8,118.4,130.3,139.8,144.8,150.9,152.3$. ESI-MS $m / z 281(\mathrm{M}+\mathrm{H})^{+}$. Anal. Calcd for $\mathrm{C}_{15} \mathrm{H}_{12} \mathrm{~N}_{4} \mathrm{O}_{2}: \mathrm{C}, 64.28 ; \mathrm{H}, 4.32 ; \mathrm{N}, 19.99$. Found: $\mathrm{C}, 64.33$; $\mathrm{H}, 4.31 ; \mathrm{N}, 20.04$.

(E)-N'-(2,4-Dihydroxybenzylidene)-1H-benzo[d]imidazole-2-carbohydrazide (6). Yield $75 \%$. Mp $>250{ }^{\circ} \mathrm{C}$ (1-PrOH). IR: 3227, 1673, 1638, $1587 \mathrm{~cm}^{-1} .{ }^{1} \mathrm{H}-\mathrm{NMR}: \delta 6.34$ (m, 2H, Ar), 6.38 (d, J = 6.5 Hz, 1H, Ar), 7.57 (m, 4H, Ar), $8.68(\mathrm{~s}, 1 \mathrm{H}, \mathrm{CH}), 10.09(\mathrm{~s}, 1 \mathrm{H}, \mathrm{OH}), 11.43(\mathrm{~s}, 1 \mathrm{H}, \mathrm{OH}), 12.59(\mathrm{~s}, 1 \mathrm{H}, \mathrm{NH}), 13.45(\mathrm{~s}, 1 \mathrm{H}, \mathrm{NH})$. ${ }^{13}$ C-NMR: $\delta$ 105.0, 109.6, 115.1, 115.9, 123.2, 125.9, 127.6, 134.7, 135.1, 137.8, 145.9, 148.0. ESI-MS m/z $297(\mathrm{M}+\mathrm{H})^{+}$. Anal. Calcd for $\mathrm{C}_{15} \mathrm{H}_{12} \mathrm{~N}_{4} \mathrm{O}_{3}: \mathrm{C}, 60.81 ; \mathrm{H}, 4.08 ; \mathrm{N}, 18.91$. Found: $\mathrm{C}, 60.76 ; \mathrm{H}, 4.10$; $\mathrm{N}, 18.94$.

(E)- $\mathrm{N}^{\prime}-\left(2,5\right.$-Dihydroxybenzylidene)-1H-benzo[d]imidazole-2-carbohydrazide (7). Yield $81 \%$. Mp $>250{ }^{\circ} \mathrm{C}$ (EtOH). IR: 3238, 1681, 1620, $1586 \mathrm{~cm}^{-1} .{ }^{1} \mathrm{H}-\mathrm{NMR}: \delta 6.77(\mathrm{~d}, J=9.0 \mathrm{~Hz}, 1 \mathrm{H}, \mathrm{Ar}), 6.79(\mathrm{~d}, J=9.0 \mathrm{~Hz}$, 1H, Ar), 6.96 (s, 1H, Ar), 7.59 (m, 4H, Ar), 8.75 (s, 1H, CH) 9.03 (s, 1H, OH), 10.38 (s, 1H, OH), 12.67 (s, 1H, NH), 13.48 (s, 1H, NH). ${ }^{13} \mathrm{C}-\mathrm{NMR}: \delta 105.7,110.3,115.6,116.0,123.3,126.5,130.2,134.9,135.6$, 138.1, 145.8, 148.1. ESI-MS $m / z 297(\mathrm{M}+\mathrm{H})^{+}$. Anal. Calcd for $\mathrm{C}_{15} \mathrm{H}_{12} \mathrm{~N}_{4} \mathrm{O}_{3}: \mathrm{C}, 60.81 ; \mathrm{H}, 4.08 ; \mathrm{N}, 18.91$. Found: $\mathrm{C}, 60.86 ; \mathrm{H}, 4.09 ; \mathrm{N}, 18.87$.

(E)- $\mathrm{N}^{\prime}$-(2,3,4-Trihydroxybenzylidene)-1H-benzo[d]imidazole-2-carbohydrazide (8). Yield $62 \%$. Mp $>250{ }^{\circ} \mathrm{C}$ (EtOH). IR: 3228, 3127, 3061, 1672, $1644 \mathrm{~cm}^{-1} .{ }^{1} \mathrm{H}-\mathrm{NMR}: \delta 6.42$ (d, J = $\left.8.5 \mathrm{~Hz}, 1 \mathrm{H}, \mathrm{Ar}\right), 6.76$ (d, J = $8.5 \mathrm{~Hz}$, 1H, Ar), $7.64(\mathrm{~m}, 4 \mathrm{H}, \mathrm{Ar}), 8.65(\mathrm{~s}, 1 \mathrm{H}, \mathrm{CH}), 9.57(\mathrm{~s}, 2 \mathrm{H}, \mathrm{OH}), 11.49$ (s, 1H, OH), $12.65(\mathrm{~s}, 1 \mathrm{H}, \mathrm{NH}), 13.48$ (s, 1H, NH). ${ }^{13} \mathrm{C}-\mathrm{NMR}: \delta 106.2,111.0,115.7,116.1,125.6,127.1,135.0,138.6,138.7,139.9,147.1,149.2$. ESI-MS $m / z 313(\mathrm{M}+\mathrm{H})^{+}$. Anal. Calcd for $\mathrm{C}_{15} \mathrm{H}_{12} \mathrm{~N}_{4} \mathrm{O}_{4}$ : $\mathrm{C}, 57.69 ; \mathrm{H}, 3.87 ; \mathrm{N}, 17.94$. Found: $\mathrm{C}, 57.64$; $\mathrm{H}, 3.88 ; \mathrm{N}, 17.91$. 
(E)- $N^{\prime}$-(2,4,6-Trihydroxybenzylidene)-1H-benzo[d]imidazole-2-carbohydrazide (9). Yield $60 \%$. Mp $>250{ }^{\circ} \mathrm{C}$ (EtOH). IR: 3225, 1672, $1592 \mathrm{~cm}^{-1} .{ }^{1} \mathrm{H}-\mathrm{NMR}: \delta 5.80$ (s, 1H, Ar) 5.86 (s, 1H, Ar), 7.26 (d, J = $7.0 \mathrm{~Hz}$, 1H, Ar), 7.30-7.78 (m, 3H, Ar), 8.98 (s, 1H, CH), 10.14 (s, 1H, OH), $11.14(\mathrm{~s}, 2 \mathrm{H}, \mathrm{OH}), 12.68(\mathrm{~s}, 1 \mathrm{H}, \mathrm{NH})$, 13.43 (s, $1 \mathrm{H}, \mathrm{NH}) .{ }^{13} \mathrm{C}-\mathrm{NMR}: \delta 99.2,103.9,119.7,127.9,138.9,140.1,145.2,147.4,151.0$. ESI-MS $\mathrm{m} / \mathrm{z} 313$ $(\mathrm{M}+\mathrm{H})^{+}$. Anal. Calcd for $\mathrm{C}_{15} \mathrm{H}_{12} \mathrm{~N}_{4} \mathrm{O}_{4}: \mathrm{C}, 57.69 ; \mathrm{H}, 3.87 ; \mathrm{N}, 17.94$. Found: $\mathrm{C}, 57.73 ; \mathrm{H}, 3.86 ; \mathrm{N}, 17.92$.

(E)-N'-(2-Hydroxy-4-methoxybenzylidene)-1H-benzo[d]imidazole-2-carbohydrazide (10). Yield 79\%. Mp $>250{ }^{\circ} \mathrm{C}$ (EtOH). IR: 3214, 1665, 1633, 1607, $1567 \mathrm{~cm}^{-1} .{ }^{1} \mathrm{H}-\mathrm{NMR}: \delta 3.79$ (s, 3H, $\left.\mathrm{OCH}_{3}\right), 6.54(\mathrm{~m}, 2 \mathrm{H}, \mathrm{Ar}), 7.48$ $(\mathrm{m}, 5 \mathrm{H}, \mathrm{Ar}), 8.74(\mathrm{~s}, 1 \mathrm{H}, \mathrm{CH}), 11.55(\mathrm{~s}, 1 \mathrm{H}, \mathrm{NH}), 12.69(\mathrm{~s}, 1 \mathrm{H}, \mathrm{OH}), 13.47(\mathrm{~s}, 1 \mathrm{H}, \mathrm{NH}) .{ }^{13} \mathrm{C}-\mathrm{NMR}: \delta 58.5$, 104.4, 109.7, 114.9, 115.8, 123.1, 126.0, 127.6, 134.5, 135.2, 137.7, 145.7, 147.6. $\delta$ ESI-MS $m / z 311(\mathrm{M}+\mathrm{H})^{+}$. Anal. Calcd for $\mathrm{C}_{16} \mathrm{H}_{14} \mathrm{~N}_{4} \mathrm{O}_{3}$ : C, 61.93; $\mathrm{H}, 4.55 ; \mathrm{N}, 18.06$. Found: $\mathrm{C}, 61.99 ; \mathrm{H}, 4.53 ; \mathrm{N}, 18.02$.

(E)-N'-(3-Ethoxy-2-hydroxybenzylidene)-1H-benzo[d]imidazole-2-carbohydrazide (11). Yield 70\%. Mp $>250{ }^{\circ} \mathrm{C}$ (EtOH). IR: 3322, 1694, 1610, $1583 \mathrm{~cm}^{-1}$. ${ }^{1} \mathrm{H}-\mathrm{NMR}: \delta 1.36\left(\mathrm{t}, J=7.0 \mathrm{~Hz}, 3 \mathrm{H}, \mathrm{CH}_{3}\right), 4.07(\mathrm{q}, J=7.0 \mathrm{~Hz}$, $\left.2 \mathrm{H}, \mathrm{CH}_{2}\right), 6.86(\mathrm{~m}, 1 \mathrm{H}, \mathrm{Ar}), 7.03(\mathrm{~d}, J=8.0 \mathrm{~Hz}, 1 \mathrm{H}, \mathrm{Ar}),(\mathrm{d}, J=8.0 \mathrm{~Hz}, 1 \mathrm{H}, \mathrm{Ar}), 7.59(\mathrm{~m}, 4 \mathrm{H}, \mathrm{Ar}), 8.84$ (s, 1H, CH), 10.99 (s, 1H, NH), $12.81(\mathrm{~s}, 1 \mathrm{H}, \mathrm{OH}), 13.53$ (s, 1H, NH). ${ }^{13} \mathrm{C}-\mathrm{NMR}: \delta$ 14.9, 65.0, 106.3, 107.7, 115.6, 115.8, 123.1, 124.5, 129.9, 135.8, 137.9, 146.2, 147.4, 149.2. ESI-MS $m / z 325(\mathrm{M}+\mathrm{H})^{+}$. Anal. Calcd for $\mathrm{C}_{17} \mathrm{H}_{16} \mathrm{~N}_{4} \mathrm{O}_{3}$ : C, 62.95; $\mathrm{H}, 4.97 ; \mathrm{N}, 17.27$. Found: $\mathrm{C}, 63.01 ; \mathrm{H}, 4.99 ; \mathrm{N}, 17.23$.

(E)- $N^{\prime}$-(3-Hydroxy-4-methoxybenzylidene)-1H-benzo[d]imidazole-2-carbohydrazide (12). Yield $71 \%$. Mp $>250{ }^{\circ} \mathrm{C}$ (EtOH). IR: 3277, 3219, 1673, 1612, $1567 \mathrm{~cm}^{-1} .{ }^{1} \mathrm{H}-\mathrm{NMR}: \delta 3.96$ (s, 3H, OCH $), 6.99$ (d, J = $8.5 \mathrm{~Hz}$, $1 \mathrm{H}, \mathrm{Ar}), 7.0(\mathrm{~d}, J=8.5 \mathrm{~Hz}, 1 \mathrm{H}, \mathrm{Ar}), 7.29(\mathrm{~s}, 1 \mathrm{H}, \mathrm{Ar}), 7.49$ (m, 4H, Ar), 8.49 (s, 1H, CH), 9.39 (br s, 1H, $\mathrm{OH}), 12.25$ (br s, $1 \mathrm{H}, \mathrm{NH}), 13.20$ (br s, $1 \mathrm{H}, \mathrm{NH}) .{ }^{13} \mathrm{C}-\mathrm{NMR}: \delta 58.5,106.0,106.3,110.1,115.9,116.7,124.8$, 128.5, 129.7, 135.4, 136.1, 145.4, 146.9. ESI-MS $m / z 311(\mathrm{M}+\mathrm{H})^{+}$. Anal. Calcd for $\mathrm{C}_{16} \mathrm{H}_{14} \mathrm{~N}_{4} \mathrm{O}_{3}$ : C, 61.93; H, 4.55; N, 18.06. Found: C, 61.88; H, 4.56; N, 18.10.

(E)-N'-(4-(Diethylamino)-2-hydroxybenzylidene)-1H-benzo[d]imidazole-2-carbohydrazide (13). Yield 78\%. $\mathrm{Mp}>250{ }^{\circ} \mathrm{C}(\mathrm{EtOH}) . \mathrm{IR}: 1668,1631,1586 \mathrm{~cm}^{-1} .{ }^{1} \mathrm{H}-\mathrm{NMR}: \delta 1.10\left(\mathrm{t}, J=7.0 \mathrm{~Hz}, 6 \mathrm{H}, \mathrm{CH}_{3}\right), 3.35$ $\left(\mathrm{q}, J=7.0 \mathrm{~Hz}, 4 \mathrm{H}, \mathrm{CH}_{2}\right), 6.12(\mathrm{~s}, 1 \mathrm{H}, \mathrm{Ar}), 6.27(\mathrm{~d}, J=6.0 \mathrm{~Hz}, 1 \mathrm{H}, \mathrm{Ar}), 7.14(\mathrm{~d}, J=6.0 \mathrm{~Hz}, 1 \mathrm{H}, \mathrm{Ar}), 7.32$ $(\mathrm{m}, 2 \mathrm{H}, \mathrm{Ar}), 7.57(\mathrm{~m}, 1 \mathrm{H}, \mathrm{Ar}), 7.77(\mathrm{~m}, 1 \mathrm{H}, \mathrm{Ar}), 8.60(\mathrm{~s}, 1 \mathrm{H}, \mathrm{CH}), 11.42(\mathrm{~s}, 1 \mathrm{H}, \mathrm{OH}), 12.52(\mathrm{~s}, 1 \mathrm{H}, \mathrm{NH})$, 13.42 (s, 1H, NH). ${ }^{13} \mathrm{C}-\mathrm{NMR}: \delta 13.2,48.9,99.2,103.6,106.7,109.9,115.9,122.7,125.9,131.4,137.7,140.0$, 142.3, 146.2, 150.6. ESI-MS $m / z 352(\mathrm{M}+\mathrm{H})^{+}$. Anal. Calcd for $\mathrm{C}_{19} \mathrm{H}_{21} \mathrm{~N}_{5} \mathrm{O}_{2}: \mathrm{C}, 64.94 ; \mathrm{H}, 6.02 ; \mathrm{N}, 19.93$. Found: C, 65.01; H, 5.99; N, 19.97.

(E)-N'-(5-Chloro-2-hydroxybenzylidene)-1H-benzo[d]imidazole-2-carbohydrazide (14). Yield $85 \% . \mathrm{Mp}>250{ }^{\circ} \mathrm{C}$ (EtOH). IR: 3215, 1680, 1605, $1591 \mathrm{~cm}^{-1} .{ }^{1} \mathrm{H}-\mathrm{NMR}: \delta 6.96$ (d, J = $\left.8.0 \mathrm{~Hz}, 1 \mathrm{H}, \mathrm{Ar}\right), 7.33-7.65$ (m, 6H, Ar), 8.82 (s, 1H, CH), 11.15 (br s, $1 \mathrm{H}, \mathrm{OH}), 12.90$ (br s, $1 \mathrm{H}, \mathrm{NH}), 13.30$ (br s, $1 \mathrm{H}, \mathrm{NH}) .{ }^{13} \mathrm{C}-\mathrm{NMR}: \delta 105.6$, $107.4,109.9,115.9,117.2,122.8,123.0,128.0,129.5,134.4,141.5,141.9,147.6,151.2$. ESI-MS $m / z 315$ $(\mathrm{M}+\mathrm{H})^{+}$. Anal. Calcd for $\mathrm{C}_{15} \mathrm{H}_{11} \mathrm{ClN}_{4} \mathrm{O}_{2}: \mathrm{C}, 57.24 ; \mathrm{H}, 3.52 ; \mathrm{N}, 17.80$. Found: $\mathrm{C}, 57.30 ; \mathrm{H}, 3.51 ; \mathrm{N}, 17.83$.

(E)-N'-(5-Bromo-2-hydroxybenzylidene)-1H-benzo[d]imidazole-2-carbohydrazide (15). Yield 82\%. Mp > $250{ }^{\circ} \mathrm{C}$ (EtOH). IR: 3200, 1680, 1602, $1588 \mathrm{~cm}^{-1} .{ }^{1} \mathrm{H}-\mathrm{NMR}: \delta 6.91$ (d, $\left.J=9.0 \mathrm{~Hz}, 1 \mathrm{H}, \mathrm{Ar}\right), 7.33$ (d, J = $6.0 \mathrm{~Hz}$, $1 \mathrm{H}, \mathrm{Ar}), 7.35(\mathrm{~d}, J=6.0 \mathrm{~Hz}, 1 \mathrm{H}, \mathrm{Ar}), 7.60(\mathrm{~m}, 4 \mathrm{H}, \mathrm{Ar}), 8.81(\mathrm{~s}, 1 \mathrm{H}, \mathrm{CH}), 11.15(\mathrm{~s}, 1 \mathrm{H}, \mathrm{OH}), 12.85(\mathrm{~s}, 1 \mathrm{H}$, $\mathrm{NH}), 13.50$ (s, 1H, NH). ${ }^{13} \mathrm{C}-\mathrm{NMR}: \delta 105.2,107.5,109.9,116.0,117.3,122.9,123.0,128.2,129.4,134.5$, 141.5, 142.0, 147.7, 151.1. ESI-MS $m / z 359(\mathrm{M}+\mathrm{H})^{+}$. Anal. Calcd for $\mathrm{C}_{15} \mathrm{H}_{11} \mathrm{BrN}_{4} \mathrm{O}_{2}$ : C, 50.16; $\mathrm{H}, 3.09$; $\mathrm{N}, 15.60$. Found: C, 50.22; H, 3.11; N, 15.57 .

(E)-N'-((2-Hydroxynaphthalen-1-yl)methylene)-1H-benzo[d]imidazole-2-carbohydrazide (16). Yield 85\%. $\mathrm{Mp}>250{ }^{\circ} \mathrm{C}$ (EtOH). IR: 3254, 1679, 1625, $1576 \mathrm{~cm}^{-1} .{ }^{1} \mathrm{H}-\mathrm{NMR}: \delta 7.26$ (d, J = $\left.9.0 \mathrm{~Hz}, 1 \mathrm{H}, \mathrm{Ar}\right), 7.35$ $(\mathrm{d}, J=6.5 \mathrm{~Hz}, 1 \mathrm{H}, \mathrm{Ar}), 7.38(\mathrm{~d}, J=6.5 \mathrm{~Hz}, 1 \mathrm{H}, \mathrm{Ar}), 7.54(\mathrm{~m}, 3 \mathrm{H}, \mathrm{Ar}), 7.82(\mathrm{~d}, J=7.0 \mathrm{~Hz}, 1 \mathrm{H}, \mathrm{Ar}), 8.11$ (m, 3H, Ar), $9.79(\mathrm{~s}, 1 \mathrm{H}, \mathrm{CH}), 12.77(\mathrm{~s}, 1 \mathrm{H}, \mathrm{NH}), 12.86(\mathrm{~s}, 1 \mathrm{H}, \mathrm{OH}), 13.58(\mathrm{~s}, 1 \mathrm{H}, \mathrm{NH}) .{ }^{13} \mathrm{C}-\mathrm{NMR}: \delta 111.6$, 
115.9, 122.0, 123.2, 123.6, 126.1, 126.8, 127.8, 131.0, 132.1, 135.0, 136.2, 137.8, 145.7, 147.3, 151.8, 158.0, 161.3. MS $m / z 331(\mathrm{M}+\mathrm{H})^{+}$. Anal. Calcd for $\mathrm{C}_{19} \mathrm{H}_{14} \mathrm{~N}_{4} \mathrm{O}_{2}$ : $\mathrm{C}, 69.08 ; \mathrm{H}, 4.27 ; \mathrm{N}, 16.96$. Found: $\mathrm{C}, 69.14$; $\mathrm{H}, 4.29 ; \mathrm{N}, 17.01$.

(E)-N'-(Naphthalen-1-ylmethylene)-1H-benzo[d]imidazole-2-carbohydrazide (19). Yield $92 \%$. Mp $>250{ }^{\circ} \mathrm{C}$ (EtOH). IR: 3208, 3044, 1618, $1558 \mathrm{~cm}^{-1} .{ }^{1} \mathrm{H}-\mathrm{NMR}: \delta 7.32$ (m, 2H, Ar), 7.65 (m, 4H, Ar), 7.81 (m, 1H, Ar), 8.01-8.16 (m, 3H, Ar), $8.74(\mathrm{~m}, 1 \mathrm{H}, \mathrm{Ar}), 9.44(\mathrm{~s}, 1 \mathrm{H}, \mathrm{CH}), 12.54(\mathrm{~s}, 1 \mathrm{H}, \mathrm{NH}), 13.55(\mathrm{~s}, 1 \mathrm{H}, \mathrm{NH}) .{ }^{13} \mathrm{C}-\mathrm{NMR}$ : $115.0,122.9,123.1,126.0,126.2,127.5,128.4,128.5,130.2,130.6,130.7,138.6,143.3,144.8,155.6$. ESI-MS $m / z 315(\mathrm{M}+\mathrm{H})^{+}$. Anal. Calcd for $\mathrm{C}_{19} \mathrm{H}_{14} \mathrm{~N}_{4} \mathrm{O}: \mathrm{C}, 72.60 ; \mathrm{H}, 4.49 ; \mathrm{N}, 17.82$. Found: $\mathrm{C}, 72.66 ; \mathrm{H}, 4.47$; $\mathrm{N}, 17.78$.

\subsection{Antiproliferative Activity}

\subsubsection{Cell Lines}

Human cervical carcinoma (HeLa), human T-lymphoblast (CEM) and mouse leukema (L1210) cells were obtained from ATCC (Middlesex, UK). Human pancreatic carcinoma (Mia-Paca 2) cells were kindly provided by Prof. Anna Karlsson (Karolinska Institute, Stockholm, Sweden). All cell lines were grown in Dulbecco's modified Eagle's medium (DMEM; Gibco, Carlsbad, CA, USA), supplemented with $10 \%$ fetal bovine serum (FBS, Gibco), $0.01 \mathrm{M}$ Hepes (Gibco) and $1 \mathrm{mM}$ sodium pyruvate (Gibco) in a humidified $5 \% \mathrm{CO}_{2}$ incubator at $37^{\circ} \mathrm{C}$.

\subsubsection{Cell Proliferation}

Suspension (L1210 and CEM cells) were seeded in 96-well microtiter plates at 60,000 cells/well in the presence of different concentrations of the compounds. The cells were allowed to proliferate for $48 \mathrm{~h}$ or $96 \mathrm{~h}$, respectively and then counted in a Coulter counter. The $50 \%$ inhibitory concentration $\left(\mathrm{IC}_{50}\right)$ was defined as the compound concentration required to reduce cell proliferation by $50 \%$. HeLa and Mia-Paca2 cells were seeded in 96-well plates at 15,000 cells/well in the presence of different concentrations of the compounds. After 4 days of incubation, the cells were trypsinized and counted in a Coulter counter.

\subsection{3. $\mathrm{IC}_{50}$ Determination}

The compounds were dissolved in DMSO at $20 \mathrm{mM}$ (stock solution) and kept in the refrigerator until use. Then, compound dilutions were made in cell culture medium, and serial compound concentrations were tested starting at $100 \mu \mathrm{M}$ as the highest concentration. The DMSO concentration, present in the highest compound concentration was $0.5 \%$ that is a concentration that did not affect the tumor cell proliferation. The $\mathrm{IC}_{50}$ values were calculated using following formula: $\mathrm{C} 1-[50-\mathrm{N} 1 \% / \mathrm{N} 2 \%-\mathrm{N} 1 \%] \times(\mathrm{C} 1-\mathrm{C} 2)$ wherein $\mathrm{C} 1$ is the compound concentration that inhibits cell proliferation more than $50 \%$; $\mathrm{C} 2$ is the compound concentration that inhibits cell proliferation less than $50 \%$; N1\% represents the cell number (in percent of control in the absence of compound) obtained in the presence of $\mathrm{C} 1$ and $\mathrm{N} 2 \%$ represents the cell number (in percent of control in the absence of compound) obtained in the presence of $\mathrm{C} 2$.

\section{Conclusions}

This study started with the aim to explore the possible antiproliferative properties of dualistic molecules bearing a combination of the hydrazone and benzimidazole moieties. Based on compounds 3-19 the in vitro activity on murine leukemia (L1210), human T-lymphoblastic leukemia (CEM), human cervix carcinoma (HeLa) and human pancreas carcinoma (Mia Paca-2) cells, we have observed that the presence of a 2-hydroxyl group on the arylidene moiety favourably modulates antiproliferative activity. In particular, hydrazones 10 and 16 inhibited the growth of all tested cell lines with low $(<10 \mu \mathrm{M})$ micromolar $\mathrm{IC}_{50}$ values. Predictions of the ADME properties for studied compounds showed 
that all hydrazones 3, 6-8, 10, 11, 13-16 might present good passive oral absorption. In conclusion by combination of benzimidazole and hydrazone pharmacophores we obtained a new class of $N^{\prime}$-(4-arylidene)-1H-benzo[d]imidazole-2-carbohydrazides endowed with significant antiproliferative activity as well as good potential absorption properties. These results further encourage us in developing an enlarged synthetic study in order to highlight SAR in this interesting class of molecules.

Acknowledgments: This study was supported by the Italian Ministero dell'Istruzione, Università e della Ricerca (PRIN 2010-2011, Prot. No. 20105YY2HL_002), the Regione Autonoma della Sardegna (Project L.R. 7/2007, No. 2012_CRP-59473) and the KU Leuven (GOA 15/19 TBA). AD gratefully acknowledges the Sardinia Regional Government for the financial support of his Ph.D. scholarship (P.O.R. Sardegna F.S.E. Operational Programme of the Autonomous Region of Sardinia, European Social Fund 2007-2013-Axis IV Human Resources, Objective 1.3, Line of Activity 1.3.1). We acknowledge Lizette van Berckelaer for excellent technical assistance.

Author Contributions: V.O., G.B., S.M. and J.B. conceived and designed the experiments; M.D., A.D., A.B., S.P. and S.L. performed the experiments; V.O., G.B., S.M. and J.B. analyzed the data; V.O. wrote the paper.

Conflicts of Interest: The authors declare no conflict of interest.

\section{Abbreviations}

The following abbreviations are used in this manuscript:

$\begin{array}{ll}\text { ADME } & \text { Adsorption, distribution, metabolism and elimination } \\ \text { IR } & \text { infrared spectra } \\ \text { NMR } & \text { Nuclear magnetic resonance } \\ \text { MS } & \text { Mass spectra } \\ \text { TPSA } & \text { Total polar surface area }\end{array}$

\section{References}

1. Yadav, G.; Ganguly, S. Structure activity relationship (SAR) study of benzimidazole scaffold for different biological activities: A mini-review. Eur. J. Med. Chem. 2015, 97, 419-443. [PubMed]

2. Gowda, N.R.; Kavitha, C.V.; Chiruvella, K.K.; Joy, O.; Rangappa, K.S.; Raghavan, S.C. Synthesis and biological evaluation of novel 1-(4-methoxyphenethyl)-1H-benzimidazole-5-carboxylic acid derivatives and their precursors as antileukemic agents. Bioorg. Med. Chem. Lett. 2009, 19, 4594-4600. [CrossRef] [PubMed]

3. Li, Y.; Tan, C.; Gao, C.; Zhang, C.; Luan, X.; Chen, X.; Liu, H.; Chen, Y.; Jiang, Y. Discovery of benzimidazole derivatives as novel multi-target EGFR, VEGFR-2 and PDGFR kinase inhibitors. Bioorg. Med. Chem. 2011, 19, 4529-4535. [CrossRef] [PubMed]

4. Determann, R.; Dreher, J.; Baumann, K.; Preu, L.; Jones, P.G.; Totzke, F.; Schächtele, C.; Kubbutat, M.H.; Kunick, C. 2-Anilino-4-(benzimidazol-2-yl)pyrimidines-A multikinase inhibitor scaffold with antiproliferative activity toward cancer cell lines. Eur. J. Med. Chem. 2012, 53, 254-263. [CrossRef] [PubMed]

5. Gao, C.; Li, B.; Zhang, B.; Sun, Q.; Li, L.; Li, X.; Chen, C.; Tan, C.; Liu, H.; Jiang, Y. Synthesis and biological evaluation of benzimidazole acridine derivatives as potential DNA-binding and apoptosis-inducing agents. Bioorg. Med. Chem. 2015, 23, 1800-1807. [CrossRef] [PubMed]

6. Guan, Q.; Han, C.; Zuo, D.; Zhai, M.; Li, Z.; Zhang, Q.; Zhai, Y.; Jiang, X.; Bao, K.; Wu, Y.; et al. Synthesis and evaluation of benzimidazole carbamates bearing indole moieties for antiproliferative and antitubulin activities. Eur. J. Med. Chem. 2014, 87, 306-315. [CrossRef] [PubMed]

7. Maji, B.; Kumar, K.; Kaulage, M.; Muniyappa, K.; Bhattacharya, S. Design and Synthesis of New Benzimidazole-Carbazole Conjugates for the Stabilization of Human Telomeric DNA, Telomerase Inhibition, and Their Selective Action on Cancer Cells. J. Med. Chem. 2014, 57, 6973-6988. [CrossRef] [PubMed]

8. Singh, M.; Tandon, V. Synthesis and biological activity of novel inhibitors of topoisomerase I: 2-Aryl-substituted 2-bis-1H-benzimidazoles. Eur. J. Med. Chem. 2011, 46, 659-669. [CrossRef] [PubMed]

9. Yoon, Y.K.; Ali, M.A.; Wei, A.C.; Shirazi, A.N.; Parang, K.; Choon, T.S. Benzimidazoles as new scaffold of sirtuin inhibitors: Green synthesis, in vitro studies, molecular docking analysis and evaluation of their anti-cancer properties. Eur. J. Med. Chem. 2014, 83, 448-454. [CrossRef] [PubMed] 
10. Yoon, Y.K.; Ali, M.A.; Wei, A.C.; Choon, T.S.; Osman, H.; Parang, K.; Shirazi, A.N. Synthesis and evaluation of novel benzimidazole derivatives as sirtuin inhibitors with antitumor activities. Bioorg. Med. Chem. 2014, 22, 703-710.

11. Salum, L.B.; Mascarello, A.; Canevarolo, R.R.; Altei, W.F.; Laranjeira, A.B.; Neuenfeldt, P.D.; Stumpf, T.R.; Chiaradia-Delatorre, L.D.; Vollmer, L.L.; Daghestani, H.N.; et al. N-(1'-naphthyl)-3,4,5trimethoxybenzohydrazide as microtubule destabilizer: Synthesis, cytotoxicity, inhibition of cell migration and in vivo activity against acute lymphoblastic leukemia. Eur. J. Med. Chem. 2015, 96, 504-518. [CrossRef] [PubMed]

12. Kaplánek, R.; Jakubek, M.; Rak, J.; Kejík, Z.; Havlík, M.; Dolenský, B.; Frydrych, I.; Hajdúch, M.; Kolář, M.; Bogdanová, K.; et al. Caffeine-hydrazones as anticancer agents with pronounced selectivity toward T-lymphoblastic leukaemia cells. Bioorg. Chem. 2015, 60, 19-29. [CrossRef] [PubMed]

13. Liao, W.; Xu, C.; Ji, X.; Hu, G.; Ren, L.; Liu, Y.; Li, R.; Gong, P.; Sun, T. Design and optimization of novel 4-(2-fluorophenoxy)quinoline derivatives bearing a hydrazone moiety as c-Met kinase inhibitors. Eur. J. Med. Chem. 2014, 87, 508-518.

14. Poti̊čková, E.; Hrušková, K.; Bureš, J.; Kovař́ková, P.; Špirková, I.A.; Pravdíková, K.; Kolbabová, L.; Hergeselová, T.; Hašková, P.; Jansová, H.; et al. Structure-activity relationships of novel salicylaldehyde isonicotinoyl hydrazone (SIH) analogs: Iron chelation, anti-oxidant and cytotoxic properties. PLoS ONE 2014, 9, e112059. [CrossRef] [PubMed]

15. Xia, Y.; Fan, C.-D.; Zhao, B.-X.; Shin, D.-S.; Miao, J.-Y. Synthesis and structure-activity relationships of novel 1-arylmethyl-3-aryl-1H-pyrazole-5-carbohydrazide hydrazone derivatives as potential agents against A549 lung cancer cells. Eur. J. Med. Chem. 2008, 43, 2347-2353. [CrossRef] [PubMed]

16. Onnis, V.; Cocco, M.T.; Fadda, R.; Congiu, C. Synthesis and evaluation of anticancer activity of 2-arylamino-6-trifluoromethyl-3-(hydrazonocarbonyl)pyridines. Bioorg. Med. Chem. 2009, 17, 6158-6165. [CrossRef] [PubMed]

17. Congiu, C.; Onnis, V. Synthesis and biological evaluation of novel acylhydrazone derivatives as potential antitumor agents. Bioorg. Med. Chem. 2013, 21, 6592-6599. [CrossRef] [PubMed]

18. Kumar, V.; Basavarajaaswamy, G.; Rai, M.V.; Poojary, B.; Pai, V.R.; Shruthi, N.; Bhat, M. Rapid 'one-pot' synthesis of a novel benzimidazole-5-carboxylate and its hydrazone derivatives as potential anti-inflammatory and antimicrobial agents. Bioorg. Med. Chem. Lett. 2015, 25, 1420-1426.

19. Liu, T.; Sun, C.; Xing, X.; Jing, L.; Tan, R.; Luo, Y.; Zhao, Y. Synthesis and evaluation of 2-[2-(phenylthiomethyl)$1 H$-benzo[d]imidazol-1-yl)acetohydrazide derivatives as antitumor agents. Bioorg. Med. Chem. Lett. 2012, 22, 3122-3125. [CrossRef] [PubMed]

20. Manfredini, S.; Vertuani, S.; Scalambra, E. Dualistic Molecules Having UV Radiation Filtrating Ability at Wide Spectrum and Potent Damping Activity of the Reactivity of Free Radicals (Radicals Scavenging). International Patent Application WO/2013/102843, 11 July 2013.

21. Lima, P.C.; Lima, L.M.; da Silva, K.C.M.; Leda, P.H.O.; de Miranda, A.L.P.; Fraga, C.A.M.; Eliezer, J.; Barreiro, E.J. Synthesis and analgesic activity of novel N-acylarylhydrazones and isosters, derived from natural safrole. Eur. J. Med. Chem. 2000, 35, 187-203. [CrossRef]

22. Lipinski, C.A.; Lombardo, F.; Dominy, B.W.; Feeney, P.J. Experimental and computational approaches to estimate solubility and permeability in drug discovery and development settings. Adv. Drug Deliv. Rev. 2001, 46, 3-26. [CrossRef]

23. Veber, D.F.; Johnson, S.R.; Cheng, H.Y.; Smith, B.R.; Ward, K.W.; Kapple, K.D. Molecular properties that influence the oral bioavailability of drug candidates. J. Med. Chem. 2002, 45, 2615-2623. [CrossRef] [PubMed]

24. Graubaum, H.; Martin, D. Reactions of benzimidazole-2-carbohydrazide with electrophilic compounds. J. Prakt. Chem. 1986, 328, 515-521. [CrossRef]

25. Harisha, R.S.; Hosamani, K.M.; Keri, R.S. Synthesis, in vitro microbial and cytotoxic studies of new benzimidazole derivatives. Arch. Pharm. 2009, 342, 412-419. [CrossRef] [PubMed]

Sample Availability: Samples of the compounds 3-19 are available from the authors.

(C) 2016 by the authors; licensee MDPI, Basel, Switzerland. This article is an open access article distributed under the terms and conditions of the Creative Commons Attribution (CC-BY) license (http:/ / creativecommons.org/licenses/by/4.0/). 